\title{
A Model for Adiabatic Mass-loss
}

\author{
Hongwei. Ge $e^{1,3}$, R. F. Webbink ${ }^{2}$ and Z. $\operatorname{Han}^{1}$ \\ ${ }^{1}$ National Astronomical Observatories/Yunnan Observatory, Chinese Academy of Sciences, \\ Kunming 650011, China; email: hongwei.ge@gmail.com \\ ${ }^{2}$ Department of Astronomy, University of Illinois, 1002 W. Green St. Urbana, IL 61801, USA \\ ${ }^{3}$ Graduate University of Chinese Academy of Sciences, Beijing 100039, China
}

\begin{abstract}
We describe our work on the development and application of a stellar structure code to compute model sequences representing donor stars in interacting binaries subject to rapid (adiabatic) mass-loss. The donor star is assumed to remain in hydrostatic equilibrium, but no heat flow is allowed. These sequences can be used to define bifurcation sequences in close binary evolution, and to circumscribe possible survivors of common envelope evolution.
\end{abstract}

Keywords. stars: evolution, stars: interiors, stars: mass loss, binaries: close

\section{Introduction}

Mass transfer is the defining feature of close binary evolution. That mass transfer typically occurs on one of three characteristic time scales. If the tidal (Roche) lobe of the donor star expands sufficiently rapidly in response to mass transfer to permit that star to remain in thermal equilibrium, mass transfer proceeds on a nuclear time scale. At the opposite extreme, mass transfer may be so rapid that the hydrostatic expansion of the donor star drives it beyond its Roche lobe, even in the absence of thermal relaxation in its interior; in this case, mass transfer proceeds on a dynamical time scale, and typically results in common envelope evolution or binary merger. In the intermediate case, donor stars stable against dynamical mass transfer, but unable to remain in thermal equilibrium, mass transfer proceeds on a thermal time scale.

We investigate the conditions for dynamical mass transfer in binary stars by constructing model sequences subject to adiabatic mass loss, that is, sequences in which the entropy (and composition) profiles are determined by the initial model of the sequence, and held fixed throughout the sequence. The initial model may be selected from any point in an evolutionary sequence for the donor star. Idealized mass loss sequences of this sort, treating donor stars as simple, compound, or centrally-condensed polytropes, were published by Hjellming \& Webbink (1987); detailed adiabatic sequences, employing full stellar models, were first calculated by Hjellming (1989a,b). Our goal is not only to explore threshold conditions for common envelope evolution in greater detail, but also to use our results to limit possible survivors of common envelope evolution, relevant to, e.g. SNe Ia, hot subdwarfs (Han et al., 2002).

\section{Adiabatic Mass-loss}

In the limit that mass loss from the donor star in an interacting binary is rapid compared to the thermal time scale of that star, the asymptotic response of that donor is adiabatic. Heat flow is negligible, but, to a good approximation, the donor remains in hydrostatic equilibrium (except very near the $L_{1}$ point). We can be treat this situation in terms of mass loss from a single star. 
We construct an evolutionary sequence of single-star models of a given mass and metallicity. Any model along this sequence may be selected as the starting model for an adiabatic mass loss sequence; that model would correspond to such a donor as it first fills its Roche lobe. The profiles in specific entropy, $s(m, X)$, and composition, $X(m)$ are held fixed along an adiabatic sequence as mass is removed from the surface. Only one other thermodynamic constraint is then needed to specify completely the state of the gas at any point in the interior of a model. That constraint is provided by the local pressure, $P(m)$, through the equation of hydrostatic equilibrium. It is sufficient, then, to solve only the equations for mass continuity and hydrostatic equilibrium, subject to the usual boundary conditions on central mass and surface optical depth, to generate an adiabatic mass loss model. The local density and temperature through the interior of the model are implicitly defined in terms of the local specific entropy, pressure, and composition.

The model solution can also be used to reconstruct the luminosity profile through the interior of a mass-losing model. Given the local state of the gas, we write the entropy gradient (2.1) in terms of the pressure and composition gradients:

$$
\mathrm{d} s / \mathrm{d} m=c_{\mathrm{P}}\left(\nabla-\nabla_{\mathrm{a}}\right) \mathrm{d} \ln P / \mathrm{d} m+\partial s / \partial X \cdot \mathrm{d} X / \mathrm{d} m .
$$

During adiabatic mass loss, $|\mathrm{d} \ln P / \mathrm{d} m|$ increases at a each mass layer, as that layer is brought toward the stellar surface, and pressure decreases. The ambient temperature gradient, $\nabla$, is therefore driven toward the adiabatic temperature gradient, $\nabla_{\mathrm{a}}$, since $\mathrm{d} s / \mathrm{d} m$ and $\mathrm{d} X / \mathrm{d} m$ are fixed. In radiative zones, the stellar luminosity at mass coordinate $m$ then follows directly from $\nabla_{\mathrm{r}}=\nabla$. In convective zones, it is necessary to invert mixinglength theory to calculate separately the contributions of radiative and convective energy fluxes, but this is straightforward algebraically. The luminosity profile provides valuable insight into the thermal relaxation of a donor star under rapid mass loss.

\section{Computing Method}

Our adiabatic mass loss code is written in FORTRAN95. It is based on Eggleton's (1971, 1972, 1973) stellar evolution code, updated as described by Han et al. (1994) and Pols et al. (1995, 1998). Initial stellar models are calculated using Evolve ZAMS (Paxton 2004), which is derived from Eggleton's code.

\section{Acknowledgements}

RFW acknowledges support from US National Science Foundation grant AST 0406726 to the University of Illinois; ZH acknowledges support from NSFC grant No. 10521001.

\section{References}

Eggleton, P. P., 1971, MNRAS 151, 351

Eggleton, P. P., 1972, MNRAS 156, 361

Eggleton, P. P., 1973, MNRAS 163, 179

Han, Z., Podsiadlowski, P., \& Eggleton, P. P. 1994, MNRAS 270, 121

Han, Z., Podsiadlowski, P., Maxted, P. F. L., Marsh, T. R., \& Ivanova, N. 2002, MNRAS 336, 449

Hjellming, M. S. 1989a, Space Sci. Revs 50, 155

Hjellming, M. S. 1989b, Ph.D. Thesis, University of Illinois at Urbana-Champaign

Hjellming, M. S. \& Webbink, R. F. 1987, ApJ 318, 794

Paxton, B. 2004, PASP 116, 699

Pols, O. R., Tout, C. A., Eggleton, P. P., \& Han, Z. 1995 MNRAS 274, 964

Pols, O. R., Schröder, K.-P., Hurley, J. R., Tout, C. A., \& Eggleton, P.P. 1998 MNRAS 298, 525 